PS1 - 176

doi:10.1017/cjn.2016.359

Where Have All the Fat Cells Gone? A Comparative Analysis of Adiposity Patterns in Childhood Brain Tumor Survivors and Non-Cancer Controls

K.W. Wang ${ }^{1,2}$, E. Kearsley ${ }^{1,2}$, N. Falzone $e^{1,2}$, A. Fleming ${ }^{1,3}, S$. Burrow $^{4}$, R.J. de Souza ${ }^{5}$, L. Thabane ${ }^{1,5,6,7,8}$, M.C. Samaan ${ }^{1,2,5}$ ${ }^{I}$ Department of Pediatrics, McMaster University, Hamilton, ON

${ }^{2}$ Division of Pediatric Endocrinology, McMaster Children's Hospital, Hamilton, $O N$

${ }^{3}$ Division of Pediatric Hematology/Oncology, McMaster

Children's Hospital, Hamilton, ON

${ }^{4}$ Division of Orthopedic Surgery, Department of Surgery,

McMaster University Medical Centre, Hamilton, ON

${ }_{5}^{5}$ Department of Clinical Epidemiology \& Biostatistics, McMaster

University, Hamilton, $\mathrm{ON}$

${ }^{6}$ Department of Anesthesia, McMaster University, Hamilton, ON

${ }^{7}$ Centre for Evaluation of Medicines, St. Joseph's Health Care,

Hamilton, $O N$

${ }^{8}$ Biostatistics Unit, St Joseph's Healthcare, Hamilton, ON

wangkw2@mcmaster.ca

Brain tumors are the most common solid tumors in children in Canada. While technological advances have increased their survival rates, survivors of childhood brain tumors (SCBT) often develop obesity, which can reduce lifespan and quality of life. While adiposity is a known factor for cardiometabolic disorders in the general population, adiposity patterns in SCBT have not been determined. This study aims to investigate how adiposity patterns differ between SCBT and noncancer controls, and how lifestyle and treatment factors may contribute to these patterns. Methods: Fifty-nine SCBT and 108 non-cancer controls were recruited from the clinics at McMaster Children's Hospital. Sociodemographic and lifestyle details were collected using standardized tools to assess diet, physical activity, and sleep. Brain tumor type, location and treatment details were obtained from medical records. Total and visceral adiposity were determined by total fat mass (FM) as well as waist-to-hip (WHR) and waist-to-height ratio (WHTR). Results: SCBT have higher total and visceral adiposity, while BMI is similar to controls. Female SCBT who received radiotherapy and/or chemotherapy have higher adiposity. A dietary pattern of white bread and fried foods with low dark bread was positively associated with adiposity. Lower physical activity levels, but not sleep durations, were associated with higher adiposity. Conclusion: SCBT have higher visceral and total adiposity than non-cancer controls. Sex, chemoradiotherapy, high fat diet, and physical inactivity, can contribute to these adiposity patterns. These results provide multiple points of entry to design interventions that reduce adiposity, and may improve long-term outcomes in SCBT.

PS1 - 182

doi:10.1017/cjn.2016.360

Epidemiology and Review of the Trends of Brain Tumors in Children under the Age of 3: A Report from the Canadian Pediatric Brain Tumour Consortium

S. Thulasiraja ${ }^{\text {h, D.L. Johnston, D. Keene }}$

${ }^{I}$ Children's Hospital of Eastern Ontario, Ottawa, ON

sthulasirajah@cheo.on.ca
To describe the epidemiology of children under age 3 diagnosed with central nervous system tumors in Canada. Case ascertainment: Retrospective observation study of children under age 3 diagnosed with neoplasm involving the central nervous system between 1990 and 2005 at 13 of the Canadian pediatric oncology centres. Results: Case ascertainment was 573 persons. Below 6 months of age at diagnosis, no gender difference was seen and the commonest location of tumor was supratentorial. Embryonal tumors were the commonest, increased head circumference and vomiting were the commonest presenting symptom and survival rates were poor. Over 6 months of age at diagnosis, male predominance occurred and commonest location of tumor was the cerebellum. The commonest tumor was astrocytic, vomiting was the commonest presenting symptom and survival was better than in the under 6month age group. Conclusion: Over the study period, the incidence rate and degree of resection remained stable. A trend to increased survival in children with ependymal tumors occurred; while, with medulloblastoma, survival decreased.

PS1 - 183

doi:10.1017/cjn.2016.361

\section{Recommendations for the Referral of Patients from Proton Beam Therapy, an Alberta Health Services Report: A Model for Canada?}

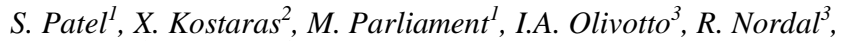
K. Aronyk ${ }^{1}$, N. Hagen ${ }^{3}$

${ }^{1}$ University of Alberta, Edmonton, $A B$

${ }^{2}$ Alberta Health Services, Calgary, $A B$

${ }^{3}$ University of Calgary, Calgary, $A B$

samir.patel2@albertahealthservices.ca

Proton beam therapy (PBT) offers compelling advantages in physical dose distribution compared to photon therapy. There are increasing numbers of gantry-based proton facilities worldwide but no such facilities exist in Canada. To access PBT, Canadian patients must travel abroad for treatment at high cost. In the face of limited access, this report seeks to provide recommendations for the selection of patients most likely to benefit from PBT and suggests an out-ofcountry referral process. METHODS: A systematic literature search for studies between January 1990 and May 2014 evaluating clinical outcomes after PBT. A draft report was developed through review of evidence, externally reviewed, and approved by the Alberta Health Services Cancer Care Proton Therapy Guidelines steering committee. RESULTS: Proton therapy is often used to treat tumours close to radiosensitive tissues, and children at risk of developing significant late effects of radiation therapy (RT). Local control rates with PBT appear similar to or, in some cases, higher than photon RT in uncontrolled and retrospective studies. Randomized trials comparing equivalent doses of PBT and photon RT are not available. SUMMARY: Referral for PBT is recommended for patients being treated with curative intent, with an expectation for long-term survival, and who are able and willing to travel abroad to a proton facility. Commonly accepted indications for referral include chordoma and chondrosarcoma, intraocular melanoma, and solid tumours in children and adolescents occurring in patients with greatest risk of long-term sequelae. Current data do not provide sufficient evidence to recommend routine referral of patients with most head and neck, breast, lung, gastrointestinal tract, and pelvic cancers including prostate cancer. It is recommended that all referrals be considered by a multidisciplinary team to select appropriate cases. 